\title{
Endorsement of the Herniasurge guidelines by the European Hernia Society
}

\author{
G. Campanelli ${ }^{1}$
}

Received: 13 July 2017 / Accepted: 20 September 2017 / Published online: 12 January 2018

(C) Springer-Verlag France SAS 2017

Inguinal hernia is one of the most common surgical diseases in the world and approximately 20 million procedures are required each year for its treatment. A mesh is used for reinforcement in most patients, making a large-scale worldwide market for these devices. The competition between companies is high, resulting in a huge number of options of different meshes and devices to be used. Evaluation of performance and in what situations to use these different devices has become so complicated that hernia surgery has almost become a "super-specialty" of its own.

To give the majority of general surgeons evidence-based guidance in their daily practice for inguinal hernia surgery, the European Hernia Society (EHS), that I have the privilege to be the current president of, published the EHS Guidelines in Hernia in 2009. It has been the most downloaded (from Hernia Springer Journal) recommendations in the recent years.

To update this work, a group of "experts" including individuals from across the globe with the participation of young researchers interested in this topic, the Herniasurge group, has further refined the EHS guidelines into these International Guidelines, now endorsed by six other international societies. The result of this massive effort can be enjoyed by general and specialized hernia surgeons around the world. It provides a useful guide in daily practice and can be used to confirm or to give education on/or evidence for techniques or meshes to be used by the individual surgeon.

It is important to emphasize that the guidelines are to be used as a support on how to make your own algorithm for

This comment refers to the article available at doi:10.1007/ s10029-017-1668-x.

G. Campanelli

giampiero.campanelli@grupposandonato.it

1 University of Insubria, Istituto Clinico Sant'Ambrogio Milano Hernia Center, Milan, Italy therapy. They should be viewed as "suggestions" rather than "Gospel" that must be followed religiously; in fact guidelines should be considered only supportive of the knowledge that inevitably must be considered from the perspective of the personal experience and "common sense" of the hernia surgeon.

A strength of these guidelines is that they not only take into account different meshes/devices and open and laparo/ endoscopic approaches, but also the perspective of hernia surgery in low income countries. They give valid guidance for education and safe techniques to be used (mainly the Lichtenstein technique). For each of these options, the guidelines analyze different variables like cost, complications, technical aspects, outcome, etc.

Considering the entire planet, the vast majority of countries do have very limited resources for hernia surgery. The surgical approach differs and a pure tissue repair might be the only option. Hernia missions have been initiated forming several projects around the globe for guidance and local education in line with the new guidelines adapted for local conditions.

Finally, I feel (and this is my surgical daily practice)"hernia surgery of today" must endorse a tailored approach to be used in terms of the patients wishes, his/her own needs, expectations, and in terms of other characteristics/conditions: risk factors, anatomy, surgeon availability and competence, and other surgical conditions. These guidelines will be a precious instrument in the "armamentarium" of surgeons in order for them to understand the anatomy, up to date knowledge of surgical procedures, and their own local limitations of resources, thus realizing this tailoring to give to patients the best opportunity for healing.

Prof. Giampiero Campanelli, President EHS.

Compliance with ethical standards

Conflict of interest The author declares that he has no conflict of interest. 